\title{
An updated reef fish checklist of the southernmost Caribbean reef system, with comments on the lionfish invasion
}

\section{Lista actualizada de los peces arrecifales del sistema arrecifal más meridional del Caribe, con notas sobre la invasión del pez león}

\author{
Camilo Escobar-Sierra ${ }^{\circledR} \square$, Viviana Márquez Velásquez ${ }^{\circledR} \square$, Rafael Menezes ${ }^{\circledR} \square$, \\ Ricardo Souza Rosa ${ }^{\circledR} \square$, Alejandro Loaiza-Santana ${ }^{\circledR} \square$
}

\begin{abstract}
Chocó-Darien is an important biogeographic realm, as it is a terrestrial biodiversity hotspot and the southern limit of the Caribbean reefs. However, to date there are no compiled data on the reef fish assemblage of this region. We provide an updated checklist of marine fishes from the Chocó-Darien reef system (Colombia), with comments on their geographic distribution and conservation status. Peer-reviewed studies, unpublished data and in situ visual censuses were surveyed to compose this checklist. A total of 212 reef fish species across 57 families were compiled, eight of which had no previously published records, one of which (Trachinotus falcatus) is recorded for the first time. The most speciose families were Labridae $(n=21)$, Gobiidae $(n=18)$ and Serranidae $(n=17)$. Fourteen threatened species were recorded, including one critically endangered (Epinephelus striatus) and two endangered (Balistes vetula and Scarus coelestinus). This study contributes to fill the knowledge gaps on the reef fish diversity of the Caribbean southern limit and raises concern on the spread of the lionfish invasion into the Chocó-Darién reef system.
\end{abstract}

Keywords. Atlantic. Biodiversity. Chocó-Darién. Neotropic. Pterois volitans.

\section{Resumen}

El Chocó-Darién es una importante área biogeográfica, pues es un hotspot de biodiversidad terrestre y el límite sur de los arrecifes del Caribe. Sin embargo, hasta la fecha no existen datos compilados actualizados sobre las especies de peces de arrecife de esta región. En este trabajo proporcionamos una lista actualizada de peces marinos del sistema arrecifal del Chocó-Darién (Colombia), con comentarios sobre su distribución geográfica y estado de conservación. Para la elaboración de esta lista se consultaron publicaciones, datos no publicados y censos visuales in situ. Se recopilaron 212 especies de peces de arrecife de 57 familias. Ocho de estas especies no tenían registros previos publicados, y una de ellas es registrada por primera vez (Trachinotus falcatus). Las familias con mayor número de especies fueron Labridae $(n=21)$, Gobiidae $(n=18)$ y Serranidae $(n=17)$. Se registraron 14 especies amenazadas, entre ellas una en peligro crítico (Epinephelus striatus) y dos en peligro (Balistes vetula y Scarus coelestinus). Este estudio contribuye a complementar las lagunas de conocimiento sobre la diversidad de los peces de arrecife del límite sur del Caribe y plantea la preocupación de la invasión del pez león en el sistema arrecifal del Chocó-Darién.

Palabras clave. Atlántico. Biodiversidad. Chocó-Darién. Neotrópico. Pterois volitans. 


\section{Introduction}

The continental coast of the southernmost portion of the Caribbean Sea, called Urabá Gulf, harbours particular ecological and geological features that differ markedly from other widely explored areas of the Caribbean (O’Dea, 2012). About 3.5 Myr ago, the area functioned as a deep ocean corridor connecting the fauna of the eastern Pacific and Caribbean Sea. After the closure of the Isthmus of Panama, two ecologically divergent areas were formed, with the Pacific side characterized by ocean-based environments and the Caribbean side dominated by coral reef ecosystems (Glynn, 1982).

This southernmost portion of the Caribbean Sea belongs to the Chocó-Darién biogeographic realm. It is regarded as a hotspot of global biodiversity, with high biological relevance for the Colombian Caribbean (Myers et al., 2000). The region encompasses large remnants of humid forest and a mosaic of coastal habitats, including riverine, estuarine and reef ecosystems (Díaz et al., 2000). Although the coastline is strongly influenced by large discharge of terrigenous sediment and freshwater from the Atrato River (Chevillot et al., 1993), fringing patch reefs flourish in such harsh conditions with a typical reef fish fauna and the largest living coral cover across the region (Díaz et al., 2000).

Despite its importance, the Chocó-Darién reef system remains poorly studied (but see Acero \& Garzón, 1987a; Reyes-Nivia et al., 2004), with a limited knowledge on its ichthyofauna when compared to other areas of the Colombian Caribbean, such as the San Andrés and Providencia archipelago (Victoria \& Gómez, 1984; Mejía \& Garzón-Ferreira, 2000), Santa Marta (Acero \& Garzón, 1987b; Acero \& Rivera, 1992; Grijalba-Bendeck et al., 2004) and Islas del Rosario archipelago (Acero \& Garzón, 1985; Delgadillo-Garzón \& Zapata-Ramírez, 2009). Like other areas of the Colombian Caribbean, the Chocó-Darién reef has shown clear signs of degradation, evidenced by the dominance of macroalgae, pollution, unregulated tourism and, more recently, the occurrence of one of the invasive species of lionfish Pterois volitans (Betancur-R. et al., 2011; Gómez-López et al., 2018).

Lionfishes ( $P$. miles and $P$. volitans) were the first non-native marine species to be established in the north-western North Atlantic to the Caribbean and Campeche Bank (Schofield, 2009). These species are considered voracious predators coupled with a high reproductive output (Côté et al., 2013). Hence, declines of native fish populations in reef systems have been correlated with the increment of their abundances (Green et al., 2012). Lionfishes have sharply dispersed across the Caribbean coral reefs after the first record at the Providencia Island, Colombia in 2008 (Betancur-R. et al., 2011). Over the last years, P. volitans has increasingly been reported at the Chocó-Darién reefs (Galvis \& Galvis, 2016; García \& Rueda 2018; Rojas-Vélez et al., 2019), which raises concern about the potential ecological outcomes in the near future.

This study provides an updated checklist of reef fish of the Chocó-Darién region, based on compiled data of visual censuses and literature, with notes on conservation status and species distribution. In addition, the current status on lionfish ( $P$. volitans) invasion is discussed here. This compilation lays foundation for supporting ongoing and future studies for conservation of reef fishes and management of marine resources and services of the region.

\section{Material and methods}

Study area. The study region is located in the western tip of the coast of the Urabá Gulf, Colombia, the southernmost portion of the Caribbean Sea. This area includes three sampling localities: Capurganá Bay $\left(8^{\circ} 38^{\prime} 13.45^{\prime \prime} \mathrm{N} ; 7^{\circ} 20^{\prime} 39.29^{\prime \prime} \mathrm{W}\right)$, El Aguacate $\left(8^{\circ} 37^{\prime} 5.86^{\prime \prime} \mathrm{N} ; 77^{\circ} 19^{\prime} 28.53^{\prime \prime} \mathrm{W}\right)$ and Sapzurro $\left(8^{\circ} 39^{\prime} 37.02^{\prime \prime} \mathrm{N} ; 7^{\circ} 21^{\prime} 40.37^{\prime \prime} \mathrm{W}\right)$ (Figure 1).

The waters of the Urabá Gulf are affected by an overload of freshwater and sediments from the Atrato River, which plays a critical role for local biogeography (Restrepo \& Kjerfve, 2004). The Chocó-Darién reef system is characterized by a mosaic of fringing reefs, mainly composed by large colonies of Siderastrea siderea (Figure 2a) and a less extensive coral cover of Porites porites, Pseudodiploria strigosa, Agaricia agaricites, Agaricia tenuifolia and Millepora complanata (Gómez-López et al., 2018).

Checklist compilation. The checklist was compiled from three sources: visual censuses in field expeditions, literature survey of peer-reviewed articles, and unpublished data (dissertations/theses).

Six visual censuses were performed, using roving diving technique (snorkeling) to quantify reef fish species richness in the Capurganá coastal reefs on April 2011 (Hill \& Wilkinson, 2004). Three fringing coastal reefs were chosen, based on a previous survey and information on reef spatial arrangement (Díaz et al., 2000). 


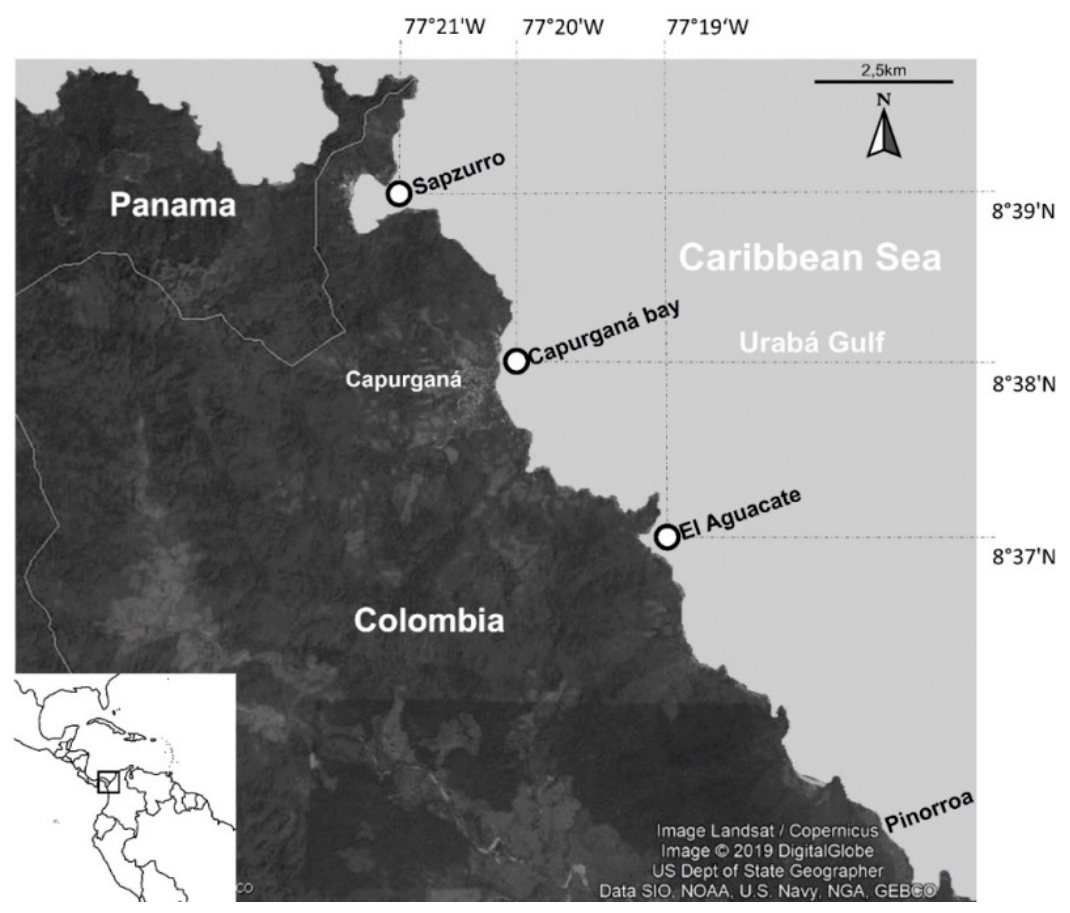

Figure 1. Western Caribbean coast of Colombia where the samplings of reef fish were carried out.

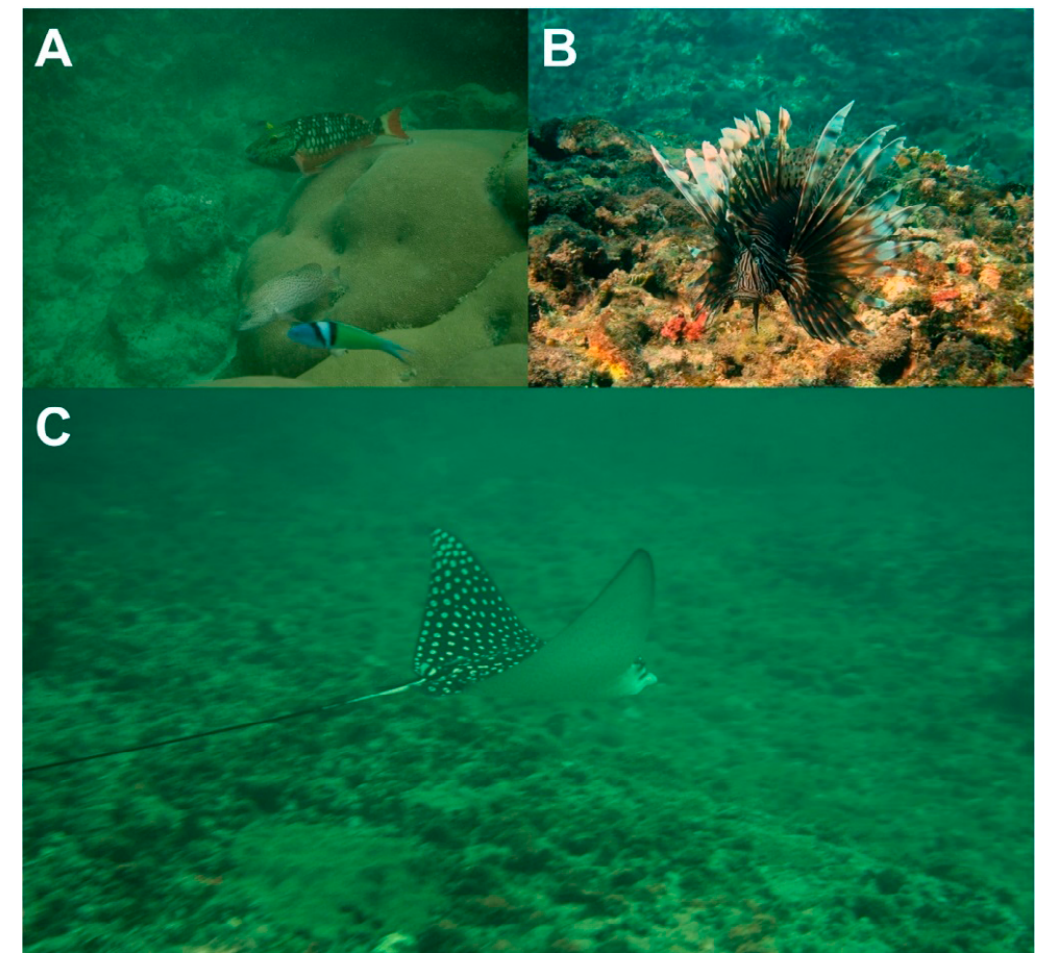

Figure 2. A small representation of the reef fish diversity recorded for the Chocó-Darién reef system, Colombia. A, Sparisoma viride, Thalassoma bifasciatum and Cephalopholis cruentata over a colony of Siderastrea siderea coral at El Aguacate; B, P. volitans at the Capurganá bay, ca. $7 \mathrm{~m}$ depth; C, Aetobatus narinari at the Capurganá bay. 
In the sampling sites, two divers freely swam for $30 \mathrm{mi}-$ nutes, identifying the fishes and, when possible, recording images and videos. The distance from the observer to the fish was a maximum of three meters, depending on the transparency, to avoid possible taxonomic errors. New records were only considered when two divers recorded the same species, or when a high-resolution image was available. Identifications of species in photos and videos were confirmed by comparisons with those provided by Reef Fish Identification Guide (Humann \& Deloach, 2003).

The list of bony fishes was organized based on Eschmeyer's Catalog of Fishes (Van der Laan et al., 2021), except for Labridae, in which Scarinae was included (Westneat \& Alfaro, 2005), whereas Weigmann (2016) was followed for elasmobranchs. Genera and species are listed in alphabetical order. For each species included in the list, we confirm the geographic distribution based on Fishbase (Froese \& Pauly, 2019). We also included the conservation status according to the Colombian National Red Book of marine fishes (Chasqui et al., 2017).

\section{Results}

Species list. A total of 212 reef fish species were compiled (Table 1), with 68 species recorded by the visual census. Eight out of the 212 species $(3.7 \%$ ) had no previous published records: Coryphopterus dicrus, Dactylopterus volitans, Diodon holocanthus, Epinephelus adscensionis, Epinephelus guttatus, Ginglymostoma cirratum, Hypanus americanus, and Trachinotus falcatus. The latter species is a new record for the area and was sighted in our visual censuses. Additionally, we report a species of the Mugil curema complex (Nirchio et al., 2017) (Table 1).

Table 1. Species of reef fishes recorded for the Chocó-Darién reef ecosystem, Colombia. References: 1, Acero \& Garzón (1987a); 2, Reyes-Nivia et al. (2004); 3, Peláez de la Torre (2010); 4, Guzmán \& Leal (2011); 5, Ramírez \& Gaviria (2013); 6, this study. Geographic range: CG, circumglobal; CT, Circumtropical; PAC, Pacific and Transatlantic (Appearing in both Western Atlantic and Eastern Atlantic); TA+MED, Transatlantic and Mediterranean;WA, Western Atlantic [Bahamas, Florida (USA), and northern Gulf of Mexico to Brazil]; WCA, Western Central Atlantic (Greater Caribbean); WI, Western Atlantic and Oceanic islands (Islands of St. Helena and Ascension). Conservation categories (IUCN 2019): CR, Critically Endangered; DD, Data deficient; EN, Endangered; LC, Least concern; NE, Not evaluated; NT, Near Threatened; VU, Vulnerable.

\begin{tabular}{|c|c|c|c|}
\hline Species & Reference & Geographic range & Conservation Status \\
\hline \multicolumn{4}{|l|}{ ORDER ORECTOLOBIFORMES } \\
\hline \multicolumn{4}{|l|}{ Family Ginglymostomatidae } \\
\hline Ginglymostoma cirratum (Bonnaterre 1788) & 3,4 & TA & VU \\
\hline \multicolumn{4}{|l|}{ ORDER MYLIOBATIFORMES } \\
\hline \multicolumn{4}{|l|}{ Family Dasyatidae } \\
\hline Hypanus americanus (Hildebrand \& Schroeder 1928) & 3,6 & WA & NT \\
\hline Styracura schmardae (Werner 1904) & 1 & WA & $\mathrm{NE}$ \\
\hline \multicolumn{4}{|l|}{ Family Myliobatidae } \\
\hline Aetobatus narinari (Euphrasen 1790) & 1,6 & TA & $\mathrm{NE}$ \\
\hline \multicolumn{4}{|l|}{ ORDER ANGUILLIFORMES } \\
\hline \multicolumn{4}{|l|}{ Family Muraenidae } \\
\hline Echidna catenata (Bloch 1795) & $1,2,3,6$ & WI & $\mathrm{NE}$ \\
\hline Enchelycore carychroa Böhlke \& Böhlke 1976 & 1 & WI & $\mathrm{NE}$ \\
\hline Enchelycore nigricans (Bonnaterre 1788) & 1 & $\mathrm{TA}$ & $\mathrm{NE}$ \\
\hline
\end{tabular}




\begin{tabular}{|c|c|c|c|}
\hline Species & Reference & Geographic range & Conservation Status \\
\hline Gymnothorax funebris Ranzani 1839 & $1,4,5$ & WA & NE \\
\hline Gymnothorax miliaris (Kaup 1856) & $1,2,3$ & TA & NE \\
\hline Gymnothorax moringa (Cuvier 1829) & $1,4,5$ & WI & NE \\
\hline Gymnothorax vicinus (Castelnau 1855) & 1 & TA & NE \\
\hline \multicolumn{4}{|l|}{ Family Chlopsidae } \\
\hline Kaupichthys hyoproroides (Strömman 1896) & 1 & $\mathrm{CT}$ & NE \\
\hline \multicolumn{4}{|l|}{ Family Ophichthidae } \\
\hline Ahlia egmontis (Jordan 1884) & 1 & WA & NE \\
\hline Myrichthys ocellatus (Lesueur 1825) & 1 & TA & NE \\
\hline \multicolumn{4}{|l|}{ Family Moringuidae } \\
\hline Moringua edwardsi (Jordan \& Bollman 1889) & 1 & WCA & NE \\
\hline \multicolumn{4}{|l|}{ ORDER CLUPEIFORMES } \\
\hline \multicolumn{4}{|l|}{ Family Engraulidae } \\
\hline Anchoa lyolepis (Evermann \& Marsh 1900) & 1 & WA & NE \\
\hline Anchoviella perfasciata (Poey 1860) & 1 & WCA & NE \\
\hline \multicolumn{4}{|l|}{ Family Clupeidae } \\
\hline Harengula clupeola (Cuvier 1829) & 1 & WA & NE \\
\hline Jenkinsia lamprotaenia (Gosse 1851) & 1 & WCA & NE \\
\hline Opisthonema oglinum (Lesueur 1818) & 1 & WA & NE \\
\hline \multicolumn{4}{|l|}{ ORDER AULOPIFORMES } \\
\hline \multicolumn{4}{|l|}{ Family Synodontidae } \\
\hline Synodus intermedius (Spix \& Agassiz 1829) & $1,2,3$ & WA & NE \\
\hline Synodus synodus (Linnaeus 1758) & 1 & TA & NE \\
\hline \multicolumn{4}{|l|}{ ORDER HOLOCENTRIFORMES } \\
\hline \multicolumn{4}{|l|}{ Family Holocentridae } \\
\hline Holocentrus adscensionis (Osbeck 1765) & $1,2,3,4,5$ & TA & NE \\
\hline Holocentrus rufus (Walbaum 1792) & $2,3,4,5$ & WA & NE \\
\hline Myripristis jacobus Cuvier 1829 & $1,2,3,4,6$ & TA & NE \\
\hline Neoniphon marianus (Cuvier 1829) & 2 & WA & NE \\
\hline Neoniphon vexillarium (Poey 1860) & $1,2,3$ & WA & NE \\
\hline Plectrypops retrospinis (Guichenot 1853) & 1 & WCA & $\mathrm{NE}$ \\
\hline \multicolumn{4}{|l|}{ ORDER OPHIDIIFORMES } \\
\hline \multicolumn{4}{|l|}{ Family Ophidiidae } \\
\hline Parophidion schmidti (Woods \& Kanazawa 1951) & 1 & WA & NE \\
\hline
\end{tabular}




\begin{tabular}{|c|c|c|c|}
\hline Species & Reference & Geographic range & Conservation Status \\
\hline \multicolumn{4}{|l|}{ Family Bythitidae } \\
\hline Ogilbia cayorum Evermann \& Kendall 1898 & 1 & WCA & NE \\
\hline \multicolumn{4}{|l|}{ ORDER SCOMBRIFORMES } \\
\hline \multicolumn{4}{|l|}{ Family Scombridae } \\
\hline Scomberomorus regalis (Bloch 1793) & 2 & TA & NE \\
\hline \multicolumn{4}{|l|}{ ORDER SYNGNATHIFORMES } \\
\hline \multicolumn{4}{|l|}{ Family Aulostomidae } \\
\hline Aulostomus maculatus Valenciennes 1841 & $1,2,3,4,6$ & WA & NE \\
\hline \multicolumn{4}{|l|}{ Family Fistulariidae } \\
\hline Fistularia tabacaria Linnaeus 1758 & 4,5 & TA & NE \\
\hline \multicolumn{4}{|l|}{ Family Syngnathidae } \\
\hline Cosmocampus brachycephalus (Poey 1868) & 1 & WA & NE \\
\hline Halicampus crinitus (Jenyns 1842) & 1 & WCA & NE \\
\hline Hippocampus reidi Ginsburg 1933 & 1 & WA & VU \\
\hline Microphis brachyurus (Bleeker 1854) & 1 & $\mathrm{CT}$ & NE \\
\hline \multicolumn{4}{|l|}{ Family Dactylopteridae } \\
\hline Dactylopterus volitans (Linnaeus 1758) & 5 & $\mathrm{TA}+\mathrm{MED}$ & $\mathrm{NE}$ \\
\hline \multicolumn{4}{|l|}{ ORDER GOBIIFORMES } \\
\hline \multicolumn{4}{|l|}{ Family Gobiidae } \\
\hline Barbulifer ceuthoecus (Jordan \& Gilbert 1884) & 1 & WA & NE \\
\hline Bathygobius soporator (Valenciennes 1837) & 1 & TA & NE \\
\hline Coryphopterus dicrus Böhlke \& Robins 1960 & 3,6 & WA & NE \\
\hline Coryphopterus eidolon Böhlke \& Robins 1960 & 2 & WCA & NE \\
\hline Coryphopterus glaucofraenum Gill 1863 & $1,2,3$ & WA & NE \\
\hline Coryphopterus lipernes Böhlke \& Robins 1962 & 2 & WCA & $\mathrm{NE}$ \\
\hline Coryphopterus personatus (Jordan \& Thompson 1905) & $2,3,6$ & WCA & $\mathrm{NE}$ \\
\hline Coryphopterus thrix Böhlke \& Robins 1960 & $2,3,6$ & WCA & NE \\
\hline Elacatinus illecebrosus (Böhlke \& Robins 1968) & $1,2,3,6$ & WCA & NE \\
\hline Elacatinus sp. & 4,5 & & \\
\hline Ginsburgellus novemlineatus (Fowler 1950) & 1 & WCA & NE \\
\hline Gnatholepis thompsoni Jordan 1904 & $1,2,3,6$ & TA & $\mathrm{NE}$ \\
\hline Lythrypnus nesiotes Böhlke \& Robins 1960 & 1 & WCA & $\mathrm{NE}$ \\
\hline Lythrypnus sp. & 1 & & \\
\hline Lythrypnus spilus Böhlke \& Robins 1960 & 1 & WCA & $\mathrm{NE}$ \\
\hline
\end{tabular}




\begin{tabular}{|c|c|c|c|}
\hline Species & Reference & Geographic range & Conservation Status \\
\hline Priolepis hipoliti (Metzelaar 1922) & 1 & WA & NE \\
\hline Tigrigobius multifasciatus (Steindachner 1876) & 1 & WCA & NE \\
\hline Tigrigobius saucrus (Robins 1960) & 2,3 & WCA & NE \\
\hline \multicolumn{4}{|l|}{ ORDER CARANGIFORMES } \\
\hline \multicolumn{4}{|l|}{ Family Sphyraenidae } \\
\hline Sphyraena barracuda (Edwards 1771) & 1,3 & CG & NT \\
\hline \multicolumn{4}{|l|}{ Family Bothidae } \\
\hline Bothus lunatus (Linnaeus 1758) & $1,2,3$ & TA & NE \\
\hline \multicolumn{4}{|l|}{ Family Carangidae } \\
\hline Caranx bartholomaei Cuvier 1833 & $1,2,3$ & WI & NE \\
\hline Caranx hippos (Linnaeus 1766) & $1,2,4$ & $\mathrm{TA}+\mathrm{MED}$ & VU \\
\hline Caranx ruber (Bloch 1793) & $1,3,4,5,6$ & WA & NE \\
\hline Decapterus sp. & 2 & & \\
\hline Trachinotus falcatus (Linnaeus 1758) & 6 & WA & NE \\
\hline \multicolumn{4}{|l|}{ ORDER BELONIFORMES } \\
\hline \multicolumn{4}{|l|}{ Family Belonidae } \\
\hline Strongylura sp. & 1,6 & & \\
\hline \multicolumn{4}{|l|}{ ORDER MUGILIFORMES } \\
\hline \multicolumn{4}{|l|}{ Family Mugilidae } \\
\hline Mugil gr. curema & 6 & CG & NE \\
\hline \multicolumn{4}{|l|}{ ORDER GOBIESOCIFORMES } \\
\hline \multicolumn{4}{|l|}{ Family Gobiesocidae } \\
\hline Acyrtops beryllinus (Hildebrand \& Ginsburg 1927) & 1 & WA & NE \\
\hline Acyrtus rubiginosus (Poey 1868) & 1 & WCA & NE \\
\hline Gobiesox punctulatus (Poey 1876) & 1 & WA & NE \\
\hline \multicolumn{4}{|l|}{ ORDER BLENNIIFORMES } \\
\hline \multicolumn{4}{|l|}{ Family Tripterygiidae } \\
\hline Enneanectes altivelis Rosenblatt 1960 & 1 & WA & $\mathrm{NE}$ \\
\hline Enneanectes boehlkei Rosenblatt 1960 & 1 & WCA & NE \\
\hline \multicolumn{4}{|l|}{ Family Labrisomidae } \\
\hline Gobioclinus kalisherae (Jordan 1904) & 1 & WA & NE \\
\hline Labrisomus nuchipinnis (Quoy \& Gaimard 1824) & 1 & TA & $\mathrm{NE}$ \\
\hline Malacoctenus macropus (Poey 1868) & 1 & WCA & NE \\
\hline Malacoctenus triangulatus Springer 1959 & 1,2 & WI & NE \\
\hline
\end{tabular}




\begin{tabular}{|c|c|c|c|}
\hline Species & Reference & Geographic range & Conservation Status \\
\hline Paraclinus nigripinnis (Steindachner 1867) & 1 & WA & NE \\
\hline Starksia variabilis Greenfield 1979 & 1 & WCA & NE \\
\hline Stathmonotus gymnodermis Springer 1955 & 1 & WA & NE \\
\hline \multicolumn{4}{|l|}{ Family Chaenopsidae } \\
\hline Acanthemblemaria rivasi Stephens 1970 & 2,3 & WCA & NE \\
\hline Coralliozetus sp. & 1 & & \\
\hline Ekemblemaria nigra (Meek \& Hildebrand 1928) & 1 & WCA & NE \\
\hline Lucayablennius zingaro (Böhlke 1957) & 2,3 & WCA & NE \\
\hline \multicolumn{4}{|l|}{ Family Dactyloscopidae } \\
\hline Dactyloscopus tridigitatus Gill 1859 & 1 & WA & $\mathrm{NE}$ \\
\hline Platygillellus rubrocinctus (Longley 1934) & 1 & WCA & NE \\
\hline \multicolumn{4}{|l|}{ Family Blenniidae } \\
\hline Entomacrodus nigricans Gill 1859 & 1 & WCA & NE \\
\hline Hypsoblennius invemar Smith-Vaniz \& Acero P. 1980 & 1 & WA & NE \\
\hline Ophioblennius macclurei (Silvester 1915) & $1,3,4,6$ & WA & NE \\
\hline \multicolumn{4}{|l|}{ ORDER ACANTHURIFORMES } \\
\hline \multicolumn{4}{|l|}{ Family Pomacanthidae } \\
\hline Holacanthus ciliaris (Linnaeus 1758) & $1,2,4$ & WI & NE \\
\hline Holacanthus tricolor (Bloch 1795) & 2,4 & WA & NE \\
\hline Pomacanthus arcuatus (Linnaeus 1758) & $1,2,3,4,6$ & WA & $\mathrm{NE}$ \\
\hline Pomacanthus paru (Bloch 1787) & $1,2,3,4,6$ & WI & NE \\
\hline \multicolumn{4}{|l|}{ Family Chaetodontidae } \\
\hline Chaetodon capistratus Linnaeus 1758 & $1,2,3,4,5,6$ & WA & NE \\
\hline Chaetodon ocellatus Bloch 1787 & $1,2,3,4,5,6$ & WA & NE \\
\hline Chaetodon sedentarius Poey 1860 & 2,6 & WA & NE \\
\hline Chaetodon striatus Linnaeus 1758 & $2,3,4,5,6$ & WI & NE \\
\hline \multicolumn{4}{|l|}{ Family Acanthuridae } \\
\hline Acanthurus chirurgus (Bloch 1787) & $1,2,3,4,5,6$ & TA & NE \\
\hline Acanthurus coeruleus Bloch \& Schneider 1801 & $1,2,3,4,5,6$ & $\mathrm{TA}$ & NE \\
\hline Acanthurus tractus Poey 1860 & $1,2,3,4,5,6$ & WCA & NE \\
\hline \multicolumn{4}{|l|}{ ORDER LOPHIIFORMES } \\
\hline Family Antennariidae & & & \\
\hline
\end{tabular}




\begin{tabular}{|c|c|c|c|}
\hline Species & Reference & Geographic range & Conservation Status \\
\hline Antennarius multiocellatus (Valenciennes 1837) & 1 & WI & NE \\
\hline \multicolumn{4}{|l|}{ ORDER TETRADONTIFORMES } \\
\hline \multicolumn{4}{|l|}{ Family Diodontidae } \\
\hline Diodon holocanthus Linnaeus 1758 & 4 & $\mathrm{CT}$ & NE \\
\hline Diodon hystrix Linnaeus 1758 & $1,2,3,6$ & $\mathrm{CT}$ & $\mathrm{NE}$ \\
\hline \multicolumn{4}{|l|}{ Family Tetraodontidae } \\
\hline Canthigaster rostrata (Bloch 1786) & $1,2,3,4,6$ & WCA & NE \\
\hline Sphoeroides spengleri (Bloch 1785) & 1 & WA & NE \\
\hline Sphoeroides testudineus (Linnaeus 1758) & 1 & WA & NE \\
\hline \multicolumn{4}{|l|}{ Family Ostraciidae } \\
\hline Acanthostracion polygonius Poey 1876 & 2,3 & WA & NE \\
\hline Acanthostracion quadricornis (Linnaeus 1758) & 2,3 & TA & NE \\
\hline Lactophrys bicaudalis (Linnaeus 1758) & $2,3,6$ & WI & NE \\
\hline Lactophrys trigonus (Linnaeus 1758) & 1 & WA & NE \\
\hline Lactophrys triqueter (Linnaeus 1758) & 2,3 & WA & NE \\
\hline \multicolumn{4}{|l|}{ Family Monacanthidae } \\
\hline Aluterus scriptus (Osbeck 1765) & 2,3 & $\mathrm{CT}$ & NE \\
\hline Cantherhines macrocerus (Hollard 1853) & $2,3,4$ & WI & NE \\
\hline Cantherhines pullus (Ranzani 1842) & $1,2,3,4,6$ & TA & NE \\
\hline Monacanthus tuckeri Bean 1906 & 2 & WCA & NE \\
\hline \multicolumn{4}{|l|}{ Family Balistidae } \\
\hline Balistes vetula Linnaeus 1758 & 2 & TA & EM \\
\hline Canthidermis sufflamen (Mitchill 1815) & $1,2,3,6$ & TA & NE \\
\hline Melichthys niger (Bloch 1786) & 2 & $\mathrm{CT}$ & NE \\
\hline \multicolumn{4}{|l|}{ ORDER CENTRARCHIFORMES } \\
\hline \multicolumn{4}{|l|}{ Family Kyphosidae } \\
\hline Kyphosus sp. & $2,3,6$ & & \\
\hline Kyphosus sectatrix (Linnaeus 1758) & $1,4,5$ & $\mathrm{TA}+\mathrm{MED}$ & NE \\
\hline \multicolumn{4}{|l|}{ Family Cirrhitidae } \\
\hline Amblycirrhitus pinos (Mowbray 1927) & 2,3 & WA & NE \\
\hline \multicolumn{4}{|l|}{ ORDER ACROPOMATIFORMES } \\
\hline Family Pempheridae & & & \\
\hline
\end{tabular}




\begin{tabular}{|c|c|c|c|}
\hline Species & Reference & Geographic range & Conservation Status \\
\hline Pempheris schomburgkii Müller \& Troschel 1848 & $1,2,3,4,5,6$ & WA & NE \\
\hline \multicolumn{4}{|l|}{ ORDER PERCIFORMES INCERTAE SEDIS } \\
\hline \multicolumn{4}{|l|}{ Family Serranidae } \\
\hline Cephalopholis cruentata (Lacepède 1802) & $1,3,4,5,6$ & WCA & $\mathrm{NE}$ \\
\hline Cephalopholis fulva (Linnaeus 1758) & 1,3 & WA & $\mathrm{NE}$ \\
\hline Epinephelus adscensionis (Osbeck 1765) & 4,5 & TA & NE \\
\hline Epinephelus guttatus (Linnaeus 1758) & 4,5 & WA & NT \\
\hline Epinephelus striatus (Bloch 1792) & $1,2,3,6$ & WA & $\mathrm{CR}$ \\
\hline Hypoplectrus nigricans (Poey 1852) & 2,3 & WCA & NE \\
\hline Hypoplectrus puella (Cuvier 1828) & $1,2,3,4,5,6$ & WCA & NE \\
\hline Hypoplectrus unicolor (Walbaum 1792) & 2,3 & WCA & NE \\
\hline Liopropoma rubre Poey 1861 & 2 & WA & NE \\
\hline Mycteroperca bonaci (Poey 1860) & 1,2 & WA & VU \\
\hline Mycteroperca tigris (Valenciennes 1833) & 2 & WA & NT \\
\hline Mycteroperca venenosa (Linnaeus 1758) & 2 & WA & VU \\
\hline Pseudogramma gregoryi (Breder 1927) & 1 & WA & NE \\
\hline Rypticus saponaceus (Bloch \& Schneider 1801) & $1,2,4,5$ & TA & $\mathrm{NE}$ \\
\hline Rypticus subbifrenatus Gill 1861 & 1 & TA & NE \\
\hline Serranus baldwini (Evermann \& Marsh 1899) & 1 & WA & NE \\
\hline Serranus tigrinus (Bloch 1790) & $1,2,3,4,5,6$ & WCA & NE \\
\hline \multicolumn{4}{|l|}{ Family Grammatidae } \\
\hline Gramma loreto Poey 1868 & $1,2,3,4,5,6$ & WA & $\mathrm{NE}$ \\
\hline Gramma melacara Böhlke \& Randall 1963 & 2 & WCA & $\mathrm{NE}$ \\
\hline \multicolumn{4}{|l|}{ Family Opistognathidae } \\
\hline Opistognathus whitehursti (Longley 1927) & 1 & WA & NE \\
\hline \multicolumn{4}{|l|}{ Family Priacanthidae } \\
\hline Heteropriacanthus cruentatus (Lacepède 1801) & 2,3 & $\mathrm{CT}$ & NE \\
\hline \multicolumn{4}{|l|}{ Family Apogonidae } \\
\hline Apogon maculatus (Poey 1860) & 1 & WA & NE \\
\hline Astrapogon puncticulatus (Poey 1867) & 1 & WA & $\mathrm{NE}$ \\
\hline Phaeoptyx conklini (Silvester 1915) & 1 & WA & NE \\
\hline Phaeoptyx pigmentaria (Poey 1860) & 1 & TA & NE \\
\hline \multicolumn{4}{|l|}{ Family Malacanthidae } \\
\hline Malacanthus plumieri (Bloch 1786) & 1 & WI & NE \\
\hline
\end{tabular}




\begin{tabular}{|c|c|c|c|}
\hline Species & Reference & Geographic range & Conservation Status \\
\hline \multicolumn{4}{|l|}{ Family Lutjanidae } \\
\hline Lutjanus analis (Cuvier 1828) & $1,2,3,6$ & WA & VU \\
\hline Lutjanus apodus (Walbaum 1792) & $2,3,4$ & WA & NE \\
\hline Lutjanus cyanopterus (Cuvier 1828) & 1,2 & WA & VU \\
\hline Lutjanus griseus (Linnaeus 1758) & 1 & WA & NE \\
\hline Lutjanus jocu (Bloch \& Schneider 1801) & $1,2,3$ & TA & DD \\
\hline Lutjanus mahogoni (Cuvier 1828) & $1,2,3,4$ & WCA & NE \\
\hline Lutjanus synagris (Linnaeus 1758) & 2,3 & WA & NE \\
\hline Ocyurus chrysurus (Bloch 1791) & $1,2,3,4,5,6$ & TA & NT \\
\hline \multicolumn{4}{|l|}{ Family Gerreidae } \\
\hline Eucinostomus sp. & 1 & & \\
\hline Gerres cinereus (Walbaum 1792) & 1 & WA & NE \\
\hline \multicolumn{4}{|l|}{ Family Haemulidae } \\
\hline Anisotremus surinamensis (Bloch 1791) & 2,3 & WA & NE \\
\hline Anisotremus virginicus (Linnaeus 1758) & $1,2,3,4,6$ & WA & NE \\
\hline Brachygenys chrysargyreum (Günther 1859) & 2,3 & WA & NE \\
\hline Haemulon album Cuvier 1830 & 2,3 & WA & $\mathrm{LC}$ \\
\hline Haemulon aurolineatum Cuvier 1830 & $1,2,3,4,6$ & WA & NE \\
\hline Haemulon bonariense Cuvier 1830 & 2,3 & WCA & NE \\
\hline Haemulon carbonarium Poey 1860 & $1,2,3,4,6$ & WCA & NE \\
\hline Haemulon flavolineatum (Desmarest 1823) & $1,2,3,4,6$ & WA & $\mathrm{NE}$ \\
\hline Haemulon macrostoma Günther 1859 & $1,2,3,4,5,6$ & WA & NE \\
\hline Haemulon parra (Desmarest 1823) & 2,3 & WA & NE \\
\hline Haemulon plumierii (Lacepède 1801) & $1,2,3,6$ & WA & NE \\
\hline Haemulon sciurus (Shaw 1803) & $2,3,4,5$ & WA & NE \\
\hline \multicolumn{4}{|l|}{ Family Sciaenidae } \\
\hline Equetus lanceolatus (Linnaeus 1758) & 2,3 & WA & NE \\
\hline Equetus punctatus (Bloch \& Schneider 1801) & $2,3,4,6$ & WA & NE \\
\hline Odontoscion dentex (Cuvier 1830) & 2,3 & WA & NE \\
\hline \multicolumn{4}{|l|}{ Family Mullidae } \\
\hline Mulloidichthys martinicus (Cuvier 1829) & $1,2,3,4,5,6$ & TA & NE \\
\hline Pseudupeneus maculatus (Bloch 1793) & $1,2,3,4,5,6$ & WA & NE \\
\hline
\end{tabular}




\begin{tabular}{|c|c|c|c|}
\hline Species & Reference & Geographic range & Conservation Status \\
\hline \multicolumn{4}{|l|}{ ORDER PERCIFORMES } \\
\hline \multicolumn{4}{|l|}{ Family Pomacentridae } \\
\hline Abudefduf saxatilis (Linnaeus 1758) & $1,2,3,4,5,6$ & $\mathrm{TA}+\mathrm{MED}$ & NE \\
\hline Abudefduf taurus (Müller \& Troschel 1848) & 1,6 & TA & NE \\
\hline Chromis cyanea (Poey 1860) & 2 & WCA & NE \\
\hline Chromis insolata (Cuvier 1830) & 2 & WCA & NE \\
\hline Chromis multilineata (Guichenot 1853) & $1,2,3,4,6$ & $\mathrm{TA}$ & NE \\
\hline Microspathodon chrysurus (Cuvier 1830) & $1,2,3,4,6$ & WA & NE \\
\hline Stegastes adustus (Troschel 1865) & $1,2,3,4,5,6$ & WCA & NE \\
\hline Stegastes diencaeus (Jordan \& Rutter 1897) & 2,3 & WCA & NE \\
\hline Stegastes leucostictus (Müller \& Troschel 1848) & $1,2,3,6$ & WA & NE \\
\hline Stegastes partitus (Poey 1868) & $1,2,3,4,6$ & WCA & NE \\
\hline Stegastes planifrons (Cuvier 1830) & $1,2,3,4,6$ & WCA & NE \\
\hline Stegastes xanthurus (Poey 1860) & $1,2,3$ & WCA & NE \\
\hline \multicolumn{4}{|l|}{ Family Labridae } \\
\hline Bodianus rufus (Linnaeus 1758) & $1,2,3,4,6$ & WA & NE \\
\hline Clepticus parrae (Bloch \& Schneider 1801) & $2,3,4$ & WCA & NE \\
\hline Doratonotus megalepis Günther 1862 & 1 & TA & NE \\
\hline Halichoeres bivittatus (Bloch 1791) & $1,2,3,4,5,6$ & WA & $\mathrm{NE}$ \\
\hline Halichoeres cyanocephalus (Bloch 1791) & 2 & WA & NE \\
\hline Halichoeres garnoti (Valenciennes 1839) & $1,2,3,4$ & WA & NE \\
\hline Halichoeres maculipinna (Müller \& Troschel 1848) & $1,2,3,4,5,6$ & WA & NE \\
\hline Halichoeres pictus (Poey 1860) & 2,3 & WCA & NE \\
\hline Halichoeres poeyi (Steindachner 1867) & $1,2,3,6$ & WA & NE \\
\hline Halichoeres radiatus (Linnaeus 1758) & $1,2,3,4,5,6$ & WI & NE \\
\hline Scarus coelestinus Valenciennes 1840 & 2 & WA & EM \\
\hline Scarus iseri (Bloch 1789) & $1,2,3,6$ & WCA & NE \\
\hline Scarus taeniopterus Lesson 1829 & 2,3 & WA & NE \\
\hline Scarus vetula Bloch \& Schneider 1801 & 2,3 & WCA & NT \\
\hline Sparisoma atomarium (Poey 1861) & 2,6 & WA & NE \\
\hline Sparisoma aurofrenatum (Valenciennes 1840) & $1,2,3,4,5,6$ & WCA & NE \\
\hline Sparisoma chrysopterum (Bloch \& Schneider 1801) & $2,3,6$ & WCA & $\mathrm{NE}$ \\
\hline
\end{tabular}




\begin{tabular}{lccc}
\hline \multicolumn{1}{c}{ Species } & Reference & Geographic range & Conservation Status \\
\hline Sparisoma radians (Valenciennes 1840) & 1 & WA & NE \\
\hline Sparisoma rubripinne (Valenciennes 1840) & $1,2,3,4,5,6$ & WA & NE \\
\hline Sparisoma viride (Bonnaterre 1788) & $1,2,3,4,5,6$ & WA & NT \\
\hline Thalassoma bifasciatum (Bloch 1791) & $1,2,3,4,5,6$ & WA & NE \\
\hline
\end{tabular}

Family Scorpaenidae

\begin{tabular}{lcll}
\hline Pterois volitans (Linnaeus 1758) & 4,6 & PAC & NE \\
\hline Scorpaena bergii Evermann \& Marsh 1900 & 1 & WA & NE \\
\hline Scorpaena grandicornis Cuvier 1829 & 1 & WA & NE \\
\hline Scorpaena isthmensis Meek \& Hildebrand 1928 & 1 & WA & NE \\
\hline Scorpaena plumieri Bloch 1789 & 1,4 & WI & NE \\
\hline Scorpaenodes caribbaeus Meek \& Hildebrand 1928 & 1 & WA & NE \\
\hline Scorpaenodes tredecimspinosus (Metzelaar 1919) & 1 & WA & NE \\
\hline
\end{tabular}

Chocó-Darién reef fishes are distributed in 21 orders and 57 families, the most representative orders being Perciformes plus Perciformes incertae sedis (43.86 \%), followed by Gobiiformes (8.49\%), Blenniiformes (8.49\%), and Tetraodontiformes $(8.02 \%)$. The most speciose families were Labridae ( $9.91 \%)$, followed by Gobiidae $(8.49 \%)$, Serranidae $(8.02 \%)$, Haemulidae and Pomacentridae $(5.66 \%)$. Likewise, the most speciose genera were Haemulon $(n=9)$, followed by Halichoeres and Lutjanus $(n=7)$, Sparisoma, Stegastes and Coryphopterus $(n=6)$.

From the recorded fishes, 96 are widespread species of the Western Atlantic, 50 of the Greater Caribbean, 33 Trans-Atlantic, seven Circumtropical, four Transatlantic and Mediterranean, two Circumglobal and one restricted to the Pacific Ocean (Figure 3). The latter species is the introduced lionfish $P$. volitans (Figure $2 b$ ).

Of the 212 species, one is listed as Data Deficient (DD), one species as Least Concern (LC), seven as Near-threatened (NT), seven as Vulnerable (VU), two as Endangered (EN) and one as Critically Endangered (CR). A total of 10 species are in a threat category for the Chocó-Darién coral reef, based in the National Red Book (Chasqui et al., 2017). The Nassau grouper (Epinephelus striatus) is
Critically Endangered. In addition, the Queen Triggerfish Balistes vetula and the Midnight Parrotfish Scarus coelestinus are categorized as Endangered. However, most species (186) have not yet been evaluated in Colombia.

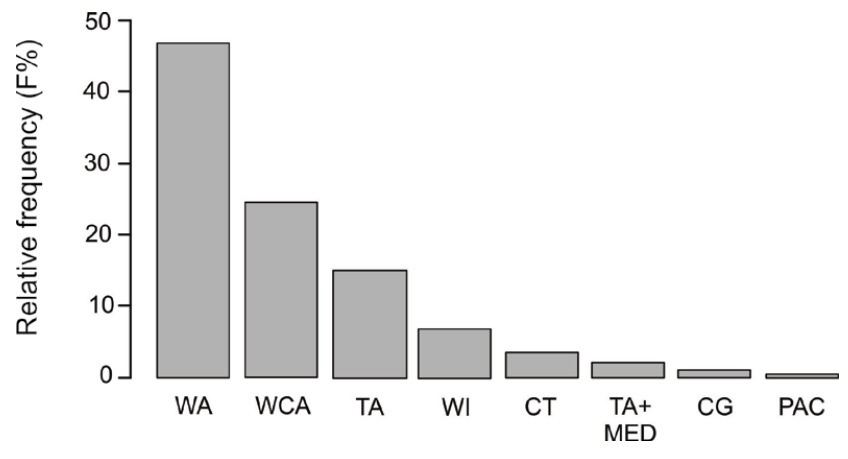

Figure 3. Relative frequency of the geographic range categories of reef fish recorded for the Chocó-Darién reef system, Colombia. CG, circumglobal; CT, circum-tropical; PAC, Pacific; TA, Transatlantic (Western Atlantic and Eastern Atlantic); TA+MED, Transatlantic and Mediterranean; WA, Western Atlantic; WCA, Western Central Atlantic (Caribbean); WI, Western Atlantic and Oceanic islands. 


\section{Discussion}

This work represents an extensive compilation on the ichthyofauna diversity of the Chocó-Darién reef system, Colombian Caribbean. Previous studies have documented contrasting number of fish species, such as 146 (Acero \& Garzón, 1987a) and 119 (Reyes-Nivia et al., 2004), which is possibly due to the use of different sampling methodologies.

The most speciose families recorded in this work (e.g., Labridae, Gobiidae, Serranidae, Haemulidae and Pomacentridae) are commonly found in the continental margins of the tropical Atlantic (Floeter, 2008). Nevertheless, the fish richness recorded for the study area (212 species) represents only $30 \%$ of the total species accounted across the Caribbean region, indicating its relatively low richness. For example, Acero \& Garzon (1987b) recorded 372 species at the Santa Marta reef systems, Colombian Caribbean, and Starck (1968) recorded 389 species at the Alligator reef, Florida Keys.

Recently, a biogeographic analysis for fish data of both reef and soft bottom divided the Caribbean region into three major provinces: (1) a central, tropical province comprising the West Indies, Bermuda and Central America; (2) a southern, upwelling-affected province spanning the entire continental shelf of northern South America; and (3) a northern, subtropical province that includes all of the Gulf of Mexico, Florida and south-eastern USA (Robertson \& Cramer, 2014). The Chocó-Darién reef system is located at the southern province covering the entire continental shelf of northern South America, holding the lowest number of fish species and percentage of local endemics $(3.4 \%)$. Likely, the particular environmental conditions of this region, such as the high loads of nutrients, low $\mathrm{pH}$, temperature and salinity variations caused by the Atrato River (McLaughlin et al., 2003, Manzello, 2010) explain, in part, the low species richness of the region.

Most of the listed species present distributions across the Western Atlantic. Biogeographically, the Western Atlantic comprises the Greater Caribbean and Brazil, with their faunas considered until recently to be partially separated by the freshwater discharge from the Amazon and Orinoco river mouths (Floeter et al., 2008). However, an extensive and diverse reef system was recently mapped for the Amazon region (called Great Amazon Reef), which represents the northern limit of the Brazilian Province and may function as an ecological corridor connecting the fauna of the Brazil and Caribbean (Francini-Filho et al., 2018). Importantly, the Caribbean shelters a higher species richness (774 species) and endemism levels (57\%) compared to Brazil (Kulbicki et al., 2013, Pinheiro et al., 2018), which, in turn, explains the high percentage of Caribbean or Western Central Atlantic endemics species in our study (23\%).

The presence of the species Gnatholepis thompsoni and Pterois volitans in the Chocó Darién region is related to different events. G. thompsoni, native from the Indian Ocean, possibly dispersed to the north Atlantic during the last interglacial period, and its expansion range has been spreading in face of climate change (Rocha et al., 2005). On the other side, P. volitans was introduced in the Florida Keys coastal waters in the 1980's, as a consequence of escapes of the aquarium trade (Morris et al., 2009). Its range has increased over the years, reaching a broad extension of the tropical and subtropical Western Atlantic and Caribbean (Schofield, 2009; Betancur-R et al., 2011). In Colombia, this lionfish was firstly reported in the oceanic islands, and subsequently in the coastal areas of the Caribbean (Betancur-R. et al., 2011). Our study raises concerns of the species' invasion at the Chocó-Darién reef system (Galvis \& Galvis, 2016; Rojas-Vélez et al., 2019), and supports the previous reports of the expansion of $P$. volitans toward southernmost portion of the Caribbean Sea. This species feeds on a wide variety of juveniles of large-bodied fish (Green \& Côté, 2014), and crustaceans, as already reported for Colombian Caribbean regions (Muñoz-Escobar \& Gil-Agudelo, 2012; Acero et al., 2019). Such feeding behavior may trigger impacts on local fish populations, and consequently, in the food web dynamics (Valdez-Moreno et al., 2012).

The present study contributes to fill up the knowledge gap on reef fish of the Chocó-Darién reef system. Further research including new technologies such as ROVs (Auster, 1997) and baited remote underwater video systems (BRUVs) (White et al., 2013) are recommended, which would enable exploring remote reef areas, such as mesophotic and rariphotic ecosystems (cf. Francini-Filho et al., 2019). Finally, in face of the fast invasion of the P. volitans and the high occurrence of threatened fishes, we recommend strengthening fish monitoring programs to subsidize management and conservation measures at the Chocó-Darién reef system. 


\section{Acknowledgments}

The authors thank Lizette Irene Quan Young for assistance and valuable comments on the first drafts of this paper. To the Universidad de Antioquia for supporting the field expedition. Thanks to Eco Hotel Playas de Capurganá and to the Echavarría family, especially Nora Ramírez and Norman Jr. Echavarría, for hosting us and facilitating the samplings. Moreover, to the many generations of marine researchers that have dedicated their time to study the most beautiful corner of the Colombian Caribbean, Capurganá.

\section{References}

Acero, A. \& Garzón, J. (1985). Peces de las islas Rosario y de San Bernardo (Colombia). I. características del área y lista de peces. Actualidades Biológicas, 14 (54), 137-148.

Acero, A. \& Garzón, J. (1987a). Los peces marinos hallados durante la expedición Urabá II al Caribe chocoano (Colombia). Anales del Instituto de Investigaciones Marinas de Punta Betín, (11), 3-1.

https://doi.org/10.25268/bimc.invemar.1987.17.0.459

Acero, A. \& Garzón, J. (1987b). Peces arrecifales de la región de Santa Marta (Caribe colombiano). I. Lista de especies y comentarios generales. Acta Biológica Colombiana, 1 (3), 83-104.

Acero, A. \& Rivera, M. (1992). Peces de las familias Chaetodontidae y Pomacanthidae en la región de Santa Marta (Colombia): densidad y relación con la calidad del arrecife. Caribbean Journal of Science, 28 (3-4), 184-190.

Acero, A., Bustos-Montes, D., Quintero, P. P., Polo-Silva, C. J. \& Muñoz, A. S. (2019). Feeding habits of Pterois volitans: a real threat to Caribbean coral reef biodiversity. In Makowski, C. \& Finkl, C. W. (Eds.). Impacts of Invasive Species on Coastal Environments. Pp. 269-314. Springer, Cham.

https:/ / doi.org/10.1007/978-3-319-91382-7_8

Auster, P. J. (1997). ROV technologies and utilization by the science community. Marine Technology Society Journal, 31 (3), 72.

Betancur-R., R., Hines, A., Acero P., A., Ortí, G., Wilbur, A. E. \& Freshwater, D. W. (2011). Reconstructing the lionfish invasion: insights into Greater Caribbean biogeography. Journal of Biogeography, 38 (7), 1281-1293.

https:/ / doi.org/10.1111/j.1365-2699.2011.02496.x

Chasqui, V., Polanco, L. A., Acero, F. A., Mejía-Falla, P. A., Navia, A., Zapata, L. A. \& Caldas, J. P. (2017).
Libro rojo de peces marinos de Colombia. Santa Marta, Colombia: Invemar. 93 pp.

Chevillot, P., Molina, A., Giraldo, L. \& Molina C. (1993). Estudio geológico e hidrológico del Golfo de Urabá. Boletín Científico CIOH, 14, 79-89.

https:/ / doi.org/10.26640/22159045.62

Côté, I. M., Green, S. J. \& Hixon, M .A. (2013). Predatory fish invaders: insights from Indo-Pacific lionfish in the western Atlantic and Caribbean. Biological Conservation, 164, 50-61.

https:/ / doi.org/10.1016/j.biocon.2013.04.014

Delgadillo-Garzón, O. \& Zapata-Ramírez, P. (2009). Evaluación rápida de peces arrecifales y su relación con la estructura del sustrato en las Islas del Rosario, área marina protegida del Caribe colombiano. Revista de la Academia Colombiana de Ciencias Exactas, Físicas y naturales, 33(127), 273-283.

Díaz, J. M., Díaz-Pulido, G. \& Sánchez, J. (2000). Distribution and structure of the southernmost Caribbean coral reefs: Golfo de Urabá, Colombia. Scientia Marina, 64, 327-336.

https:// doi.org/10.3989/scimar.2000.64n3327

Floeter, S. R., Rocha, L. A., Robertson, D. R., Joyeux, J. C., Smith-Vaniz, W. F., Wirtz, P. \& Brito, A. (2008). Atlantic reef fish biogeography and evolution. Journal of Biogeography, 35 (1), 22-47.

Francini-Filho, R. B., Asp, N. E., Siegle, E., Hocevar, J., Lowyck, K., D'Avila, N. \& Thompson, C. C. (2018). Perspectives on the Great Amazon Reef: Extension, biodiversity, and threats. Frontiers in Marine Science, 5,142 .

https:// doi.org/10.3389/fmars.2018.00142

Francini-Filho, R. B., Márquez Velázquez V., Barbosa da Silva M., Rogerio Rosa M., Gomes Sumida P. Y., Tercio Pinheiro H., Alves Rocha L., Leite Ferreira C. E., Bezerra Francini C. L. \& de Souza Rosa, R. (2019). Brazil. In: Loya Y., Puglise K., Bridge T. (Eds) Mesophotic Coral Ecosystems. Coral Reefs of the World, vol 12. Springer, Cham, pp. 163-198.

https:/ / doi.org/10.1007/978-3-319-92735-0_10

Froese, R. \& Pauly D. Editors. 2019. FishBase. World Wide Web electronic publication. Available from:

https:/ / www.fishbase.org, (accessed 01 February 2019)

Galvis, N. H. \& Galvis, R. H. (2016). Colombian citizen science to improve coral reef conservation. In: Birkeland C., Coles S. I. \& Spies N. P. (Eds.) Proceedings of 13th International Coral Reef Symposium. Pp: 606-619. Honolulu, Hawaii.

García, C. B. \& Rueda, M. (2018). The lionfish Pterois volitans (Scorpaeniformes: Scorpaenidae) invades soft-bottoms: evidence from survey data. Pan-American Journal of Aquatic Sciences, 13(3), 211-215. 
Glynn, P. W. (1982). Coral communities and their modifications relative to past and prospective Central American seaways. Advances in Marine Biology, 19, 91-132.

https:/ / doi.org/10.1016/S0065-2881(08)60087-5

Gómez-López, D. I., Acosta-Chaparro A., Gonzalez J. D., Sánchez L., Navas-Camacho, R. \& Alonso, D. (2018). Reporte del estado de los arrecifes coralinos y pastos marinos en Colombia (2016-2017). Santa Marta, Colombia: Invemar. 100 pp.

https:/ / doi.org/10.3391/ai.2009.4.3.12

Green, S. J. \& Côté, I. M. (2014). Trait-based diet selection: prey behaviour and morphology predict vulnerability to predation in reef fish communities. Journal of Animal Ecology, 83 (6), 1451-1460.

https: / / doi.org/10.1111/1365-2656.12250

Green, S. J., Akins, J. L., Maljković, A. \& Côté, I. M. (2012). Invasive lionfish drive Atlantic coral reef fish declines. PloS one, 7(3), e32596.

https:/ / doi.org/10.1371/journal.pone.0032596

Grijalba-Bendeck, M., Castañeda-Moya, E. \& Acero, A. (2004). Estructura de un ensamblaje íctico asociado a fondos duros en el Caribe colombiano empleando la técnica del censo visual estacionario (CVE). Actualidades Biológicas, 26(81), 197-211.

Guzmán, G. R. \& Leal, J. (2011). Lista de peces asociados a formaciones coralinas en la bahía Capurganá (Chocó) y algunos descriptores de su estructura comunitaria. (Trabajo de grado). Turbo, Antioquia: Programa de Ecología de Zonas Costeras, Corporación Académica Ambiental, Universidad de Antioquia. 26 pp.

Hill, J. \& Wilkinson, C. (2004). Methods for ecological monitoring of coral reefs. Townsville: Australian Institute of Marine Science. 117 pp.

Humann, P. \& Deloach, N. (2003). Reeffish identification: Florida, Caribbean, Bahamas. Florida: New World Publications. $481 \mathrm{pp}$.

Kulbicki, M., Parravicini, V., Bellwood, D. R., Arias-González, E., Chabanet, P., Floeter, S. R. \& Mouillot, D. (2013). Global biogeography of reef fishes: a hierarchical quantitative delineation of regions. PLoS One, 8(12), e81847.

https:/ / doi.org/10.1371/journal.pone.0081847

Manzello, D. P. (2010). Coral growth with thermal stress and ocean acidification: lessons from the eastern tropical Pacific. Coral reefs, 29(3), 749-758.

https:/ / doi.org/10.1007/ s00338-010-0623-4

McLaughlin, C. J., Smith, C. A., Buddemeier, R. W., Bartley, J. D. \& Maxwell, B. A. (2003). Rivers, runoff, and reefs. Global and Planetary Change, 39(1-2), 191-199.

https:/ / doi.org/10.1016/S0921-8181(03)00024-9
Mejía, L. S. \& Garzón-Ferreira, J. (2000). Estructura de comunidades de peces arrecifales en cuatro atolones del Archipiélago de San Andrés y Providencia (Caribe suroccidental). Revista de Biología Tropical, 48 (4), 883-896.

Morris, J. A., Akins, J. L., Barse, A., Cerino, D., Freshwater, D. W., Green, S. J. \& Whitfield, P. E. (2009). Biology and ecology of the invasive lionfishes, Pterois miles and Pterois volitans. Marathon F.L. Gulf and Caribbean Fisheries Institute. 61 pp.

Muñoz-Escobar, L. \& Gil-Agudelo, D. L. (2012). Dietary composition of the lionfish, Pterois volitans (pisces: scorpaenidae), in Santa Marta and Tayrona national park. Boletin de Investigaciones Marinas y Costeras-INVEMAR, 41(2), 471-477.

Myers, N., Mittermeier, R. A., Mittermeier, C. G., da Fonseca A. B. \& Kent, J. (2000). Biodiversity hotspots for conservation priorities. Nature, 403, 853-858.

https:// doi.org/10.1038/35002501

Nirchio, M., Oliveira, C., Siccha-Ramírez, Z. R., de Sene, V. F., Sola, L., Milana, V. \& Rossi, A. R. (2017). The Mugil curema species complex (Pisces, Mugilidae): a new karyotype for the Pacific white mullet mitochondrial lineage. Comparative cytogenetics, 11(2), 225-237.

O’Dea, A., Hoyos, N., Rodríguez, F., Degracia, B. \& De Gracia, C. (2012). History of upwelling in the tropical Eastern Pacific and the paleogeography of the Isthmus of Panama. Palaeogeography Palaeoclimatology Palaeoecology, 348-349, 59-66.

https:/ / doi.org/10.1016/j.palaeo.2012.06.007

Peláez de la Torre, S. (2010). Comparación temporal de la estructura de dos comunidades ícticas de arrecifes someros en las bahías de Capurganá y en la ensenada del aguacate Caribe-Chocó. (Trabajo de grado). Bogotá D.C.: Pontificia Universidad Javeriana, Facultad de Ciencias, Departamento de Biología. 65 pp.

Pinheiro, H. T., Rocha, L. A., Macieira, R. M., ..., Floeter, S. R. (2018). South-western Atlantic reef fishes: Zoogeographical patterns and ecological drivers reveal a secondary biodiversity centre in the Atlantic Ocean. Diversity and Distributions, 24(7), 951-965.

Ramírez J. J. \& Gaviria G. (2013). Catálogo de peces asociados a los arrecifes de coral de bahía Capurganá-Choco (Colombia), una aproximación a su conocimiento. (Trabajo de grado). Turbo, Antioquia: Programa de Ecología de Zonas Costeras, Corporación Académica Ambiental, Universidad de Antioquia. 123 pp.

Restrepo, J. D. \& Kjerfve, B. (2004). The Pacific and Caribbean rivers of Colombia: water discharge, sediment transport and dissolved loads. In de Lacerda, 
L. D., Santelli, R. E., Duursma, E. K. \& Abrao, J. J. (Eds.), Environmental geochemistry in tropical and subtropical environments. Pp. 169-187. Berlin, Heidelberg: Springer.

https:/ / doi.org/10.1007/978-3-662-07060-4_14

Reyes-Nivia, M. C., Rodríguez-Ramírez, A. \& Garzón-Ferreira, J. (2004). Peces asociados a formaciones coralinas de cinco áreas del Caribe colombiano: listado de especies y primeros registros para las áreas. Boletín de Investigaciones Marinas y Costeras-INVEMAR, 33(1), 101-115.

https:/ / doi.org/10.25268/bimc.invemar.2004.33.0.250

Robertson, D. R. \& Cramer, K. L. (2014). Defining and dividing the greater Caribbean: insights from the biogeography of shore fishes. PLoS One, 9 (7), e102918.

https:/ / doi.org/10.1371/journal.pone.0102918

Rocha, L. A., Robertson, D. R., Rocha, C. R., Van Tassell, J. L., Craig, M. T. \& Bowen, B. W. (2005). Recent invasion of the tropical Atlantic by an Indo-Pacific coral reef fish. Molecular Ecology, 14 (13), 3921-3928. https:/ / doi.org/10.1111/j.1365-294X.2005.02698.x

Rojas-Vélez, S., Tavera, J. \& Acero, A. (2019). Unraveling lionfish invasion: Is Pterois volitans truly a morphologically novel predator in the Caribbean? Biological Invasions, 21(6), 1921-1931.

https:/ / doi.org/10.1007/s10530-019-01946-6

Schofield, P. J. (2009). Geographic extent and chronology of the invasion of non-native lionfish (Pterois volitans [Linnaeus 1758] and P. miles [Bennett 1828]) in the Western North Atlantic and Caribbean Sea. Aquatic Invasions, 4, 473-479.

https:/ / doi.org/10.3391/ai.2009.4.3.5
Starck, W. A. (1968). A list of fishes of Alligator Reef, Florida with comments on the nature of the Florida reef fish fauna. Undersea Biology, 1(1), 4-40.

Valdez-Moreno, M., Quintal-Lizama, C., Gómez- Lozano, R. \& del Carmen García-Rivas, M. (2012). Monitoring an alien invasion: DNA barcoding and the identification of lionfish and their prey on coral reefs of the Mexican Caribbean. PloS one, 7(6), e36636.

https:// doi.org/10.1371/journal.pone.0036636

Van der Laan, R., Fricke, R. \& Eschmeyer, W. N. (eds). (2021). Catalogue of fishes: classification. California Academy of Sciences. Available from:

http:/ / www.calacademy.org/ scientists/ catalog-of-fishes-classification/ (Accessed 10 Feb 2021).

Victoria, P. \& Gómez, D. P. (1984). Nuevos registros de peces para la isla de San Andrés (mar Caribe de Colombia). Anales del Instituto de Investigaciones Marinas de Punta Betín, 14, 115-132.

Weigmann, S. (2016). Annotated checklist of the living sharks, batoids and chimaeras (Chondrichthyes) of the world, with a focus on biogeographical diversity Journal of Fish Biology, 88(3), 837-1037.

https:// doi.org/10.1111/jfb.12874

Westneat, M. W. \& Alfaro, M. E. (2005) Phylogenetic relationships and evolutionary history of the reef fish family Labridae. Molecular Phylogenetics and Evoltuion, 36, 370-390.

White, J., Simpfendorfer, C. A., Tobin, A. J. \& Heupel, M. R. (2013). Application of baited remote underwater video surveys to quantify spatial distribution of elasmobranchs at an ecosystem scale. Journal of Experimental Marine Biology and Ecology, 448, 281-288.

https:// doi.org/10.1016/j.jembe.2013.08.004 


\section{Camilo Escobar-Sierra}

Universidad de Antioquia.

Medellín, Colombia.

https:/ / orcid.org/0000-0001-9105-4378

camilo.escobar@udea.edu.co

Autor para correspondencia

\section{Viviana Márquez Velásquez}

Universidade Federal da Paraíba.

João Pessoa, Brasil.

https:/ / orcid.org/0000-0002-1205-7720

vmarquez@squalus.org

\section{Rafael Menezes}

Universidade Federal da Paraíba.

João Pessoa, Brasil.

https:/ / orcid.org/0000-0003-2378-3805

rafaelmenez@gmail.com

\section{Ricardo Souza Rosa}

Universidade Federal da Paraíba.

João Pessoa, Brasil.

https:/ / orcid.org/0000-0002-4289-2241

rsrosa@dse.ufpb.br

\footnotetext{
Alejandro Loaiza-Santana

Universidad de Antioquia.

Medellín, Colombia.

https:/ / orcid.org/0000-0003-2608-8218

alejandro.loaiza.san@gmail.com
}

An updated reef fish checklist of the southernmost Caribbean reef system, with comments on the lionfish invasion

Citación del artículo: Escobar-Sierra, C., Márquez-Velásquez, V., Menezes, R., Souza-Rosa, R. Loaiza-Santana, A. (2021). An updated reef fish checklist of the southernmost Caribbean reef system, with comments on the lionfish invasion. Biota Colombiana, 22(2), 70-87.

https: / / doi.org/10.21068/c2021.v22n02a04

Recibido: 27 de septiembre 2020

Aceptado: 2 de marzo 2021 